\title{
Piscina Temperada con Energía Solar
}

\section{RESUMEN}

El artículo trata sobre los detalles técnicos para la construcción de una piscina temperada con colectores solares, en su construcción se utilizó la madera tornillo y la tecnología CPC (Colector Parabólico Compuesto). En las conexiones se procuró simplificar los caminos externos, para disminuir las pérdidas de calor se prepararon 8 cajas colectoras con reflectores de láminas de aluminio brillante; a fin de presentar una alternativa económica y ecológica para los usuarios.

Palabras Claves: Piscina temperada. Colectores solares. Reflectores de aluminio.

Temperate Pool with the Sun Energy ABSTRACT

This article deals with the technical details for the building of a temperate pool with Sun collectors. The so called "tornillo" timber and the CPC Technology (composite parabolic collector) were used for. In the coonections, was to simplify outer ways intent to diminish heat loss. Eight collecting boxes were used with reflectors of shining aluminum foils were used. The purpose is to show a cheaper economical and ecological alternative for users.

Key words: Temperate pool. Sun collectors. Aluminum reflectors.

(1) Doctor en Ingeniería Eléctrica. Actualmente Director de la Unidad de Postgrado de la Facultad de Ingeniería Electrónica, UNMSM

E-mail: wpl39@yahoo.com

\section{INTRODUCCIÓN}

Si se tiene en cuenta que el área de Colectores Solares necesaria para temperar una piscina es aproximadamente igual al $60 \%$ del área de la piscina, para el caso de usar colectores planos tradicionales su costo sería prohibitivo. El hecho de que el agua siempre estará a menos de $30^{\circ} \mathrm{C}$, permite economizar, ya que no se preocupará de aislar térmicamente los colectores; así, en Norteamérica se usa el plástico polipropileno que es barato se pinta de negro y se pone al sol sin ninguna cobertura, pero todo plástico es atacado por los rayos ultravioleta del sol y es dudosa la cantidad de años que durará este plástico.

Por otro lado, este plástico se tendría que importar si se quisiera utilizar, el volumen y peso de lo que se necesita del plástico, haría este producto caro puesto aquí en el Perú. El no tener que preocuparse del aislamiento térmico, permite, hacer los colectores de madera tornillo que no se apolilla y puede estar años a la intemperie sin deteriorarse. El cobre ha subido considerablemente por lo que es más económico ahora usar el Colector Parabólico Compuesto (CPC) que con reflectores parabólicos concentra la luz del sol en una superficie menor de cobre pintado de negro, con lo que se utilizará también menos pintura negra, que si es selectiva, se tiene que importar y es cara.

\section{FUNDAMENTO DEL CPC}

El colector parabólico compuesto (CPC) está compuesto de 2 superficies parabólicas que tienen como foco el mismo eje lo que permite concentrar en el eje luz que llegue al CPC con una inclinación menor que su ángulo de aceptación, se determina un ángulo de aceptación conveniente o sea que permite captar en el eje la mayor cantidad de la luz incidente, combinando adecuadamente diámetro del eje y profundidad de CPC.

En el eje común del CPC se constituye un tubo de cobre pintado de negro para que el tubo se caliente con la luz en el concentrada, en su interior circula el agua a ser calentada, la que se hace circular por los tubos mediante una bomba. Con la finalidad de aumentar la cantidad de luz captada que viene con cualquier inclinación es decir luz difusa, se suelda a los tubos de cobre unas aletas convenientemente dispuestas las que también se pintan de negro para convertir en calor la luz incidente (ver Figura 1).

EI CPC fue desarrollado en los Reactores Nucleares para captar le energía de los neutrones que vienen con cualquier dirección. 


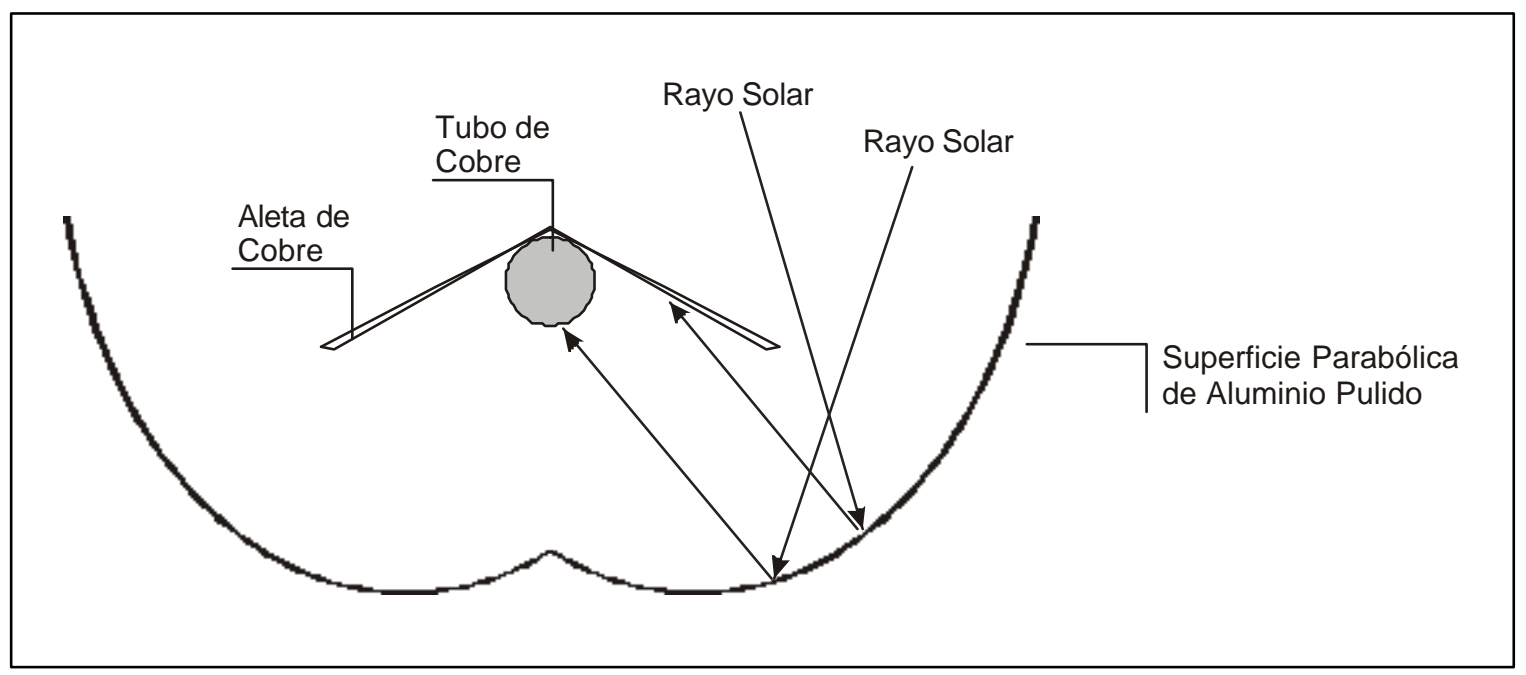

Figura 1. Colectores Parabólicos Compuestos

\section{DESCRIPCIÓN DEL SISTEMA}

El sistema de calentamiento está constituido de 2 hileras de cajas colectoras en cada hilera existen 4 cajas en paralelo que es el máximo recomendado para poner en paralelo [1] (ver Figura 2).

Estos colectores que se muestran en la Figura 2 corresponden a una pequeña piscina. La piscina tiene un área de 13,6 $\mathrm{m}^{2}$ para lo cual se colocó 8 cajas colectoras dispuestos en 2 hileras con 1,25 m de area de cada colector lo que hace un area efectiva de $10 m^{2}$.

Cada caja colectora tiene 3 tubos de cobre con sus respectivos aletas pintadas de negro mate en las que se concentra la luz del sol mediante reflectores parabólicos (CPC) de aluminio brillante, el que refleja

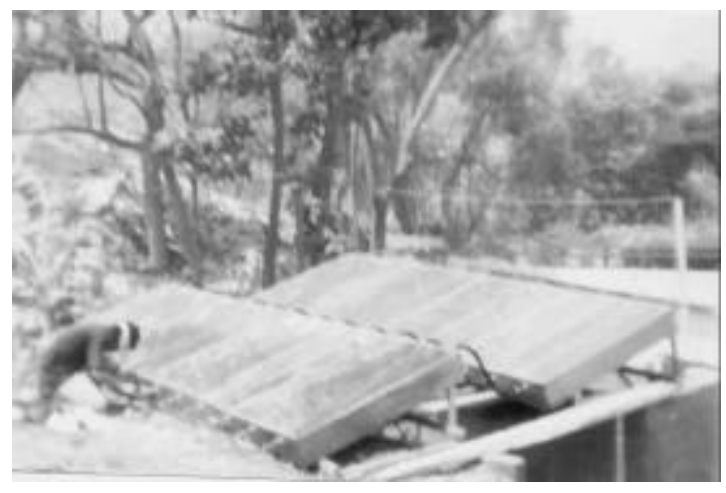

Figura 2. Colectores solares como un espejo la luz solar hacia los tubos y sus aletas teniendo un angulo de aceptación de casi $90^{\circ}$ lo que permite recibir la luz del sol en cualquier posición en que se encuentre y también luz difusa característica del CPC. El agua fría ingresa por un tubo distribuidor de cobre de 1 pulgada que manda el agua a los 12 tubos de 3/4" de la primera hilera de cajas (ver Figura 3). Cada tubo está conectado con el tubo correspondiente de la segunda hilera de cajas mediante mangueras y existe también un tubo de cobre recolector de la segunda hilera por el cual circula el agua ya calentada. La entrada de agua fría a los colectores tiene un filtro que impide que suciedad de la piscina se concentre en los colectores.

Una válvula checkse instala en linea hacia los colectores para que no regrese suciedad acumulada en el filtro hacía la piscina [2].

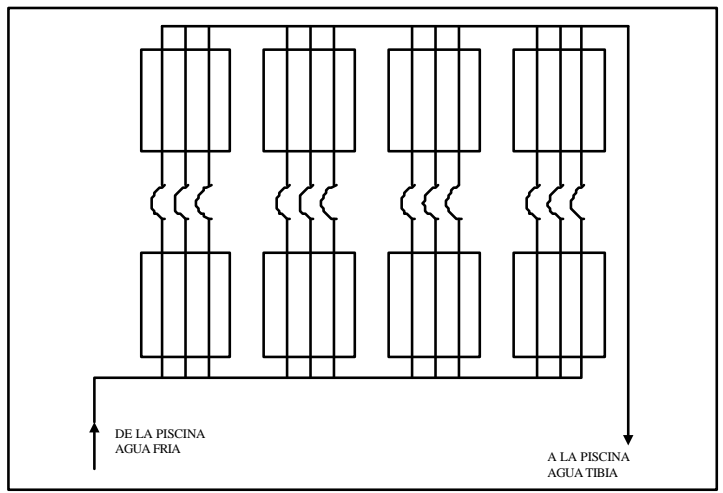

Figura 3. Esquema de conexión 


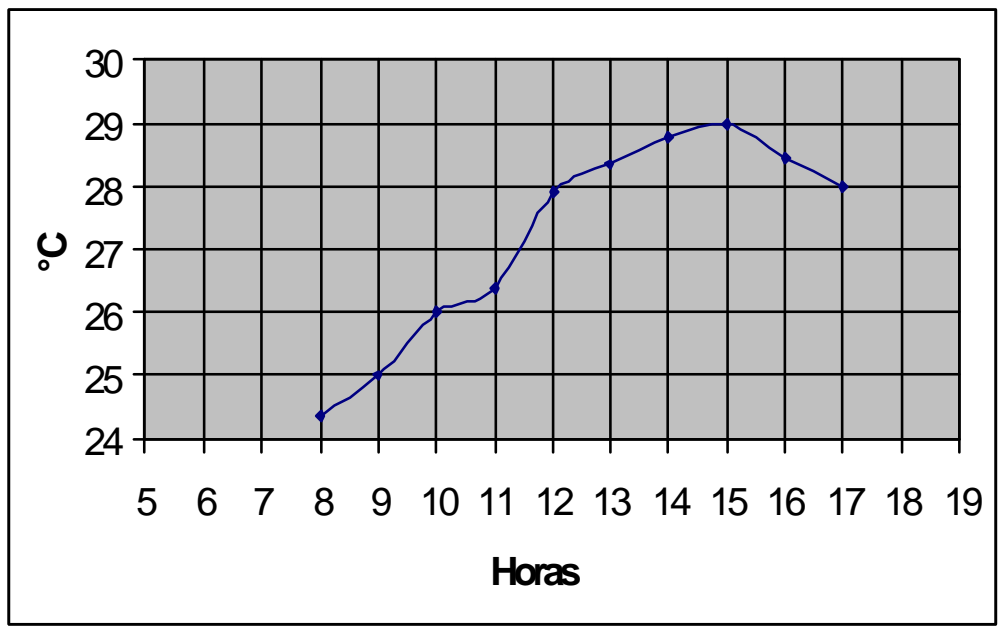

Figura 4. Evolución de la emperatura del agua en la piscina

La temperatura adecuada del agua de la piscina es entre 24 y $27^{\circ} \mathrm{C}$ [2], aquí en el Perú en Yangas (Km 57 de la carretera a Canta) donde está ubicada la piscina, ésta puede alcanzar la temperatura de $29 \stackrel{\circ}{ } \mathrm{C}$ que es una temperatura muy agradable para bañarse (ver Figura 4).

\section{CONCLUSIONES}

La eficiencia que alcanza el sistema es muy bueno ya que se alcanza hasta $29^{\circ} \mathrm{C}$ pero se debe tener cuidado de purgar el aire que puede ingresar a los tubos cuando se apaga la bomba y el agua de los tubos cae en la piscina, entrando aire en su lugar.

La purga del aire se efectúa con purgadores que son tubos de una altura conveniente conectados en la par- te superior de los tubos, por donde se expulsa el aire retenido en los tubos. Si este aire no se expulsa, no deja circular agua por el tubo para llevarse el calor producido y entonces el tubo se recalienta a altas temperaturas y eso hace ineficiente al sistema, lo ideal es que no hayan partes calientes, todos los tubos deben tener temperaturas menores a los $30^{\circ} \mathrm{C}$.

\section{BIBLIOGRAFÍA}

1. Perez Yebeña, Eduardo; Cruz Costa, Jorge. (2000). Conversão Térmica da Energía Solar. 1 ra. Edición, Edit. SPES DER/INETI. Portugal.

2. Lunde, Peter. (1980). Solar Thermal Engineering. 1 ra. Edición, Edit. John Wiley \& Sons. New York, USA. 